\title{
The efficacy of an association of palmitoylethanolamide and alpha-lipoic acid in patients with chronic prostatitis/chronic pelvic pain syndrome: A randomized clinical trial
}

\author{
Bruno Giammusso $^{1}$, Rosaria Di Mauro ${ }^{2}$, Renato Bernardini ${ }^{2}$ \\ ${ }^{1}$ Policlinico Morgagni, Catania, Italy; \\ ${ }^{2}$ BIOMETEC, Section of Pharmacology, University of Catania School of Medicine, Catania, Italy.
}

\begin{abstract}
Summary Background: Chronic prostatitis/chronic pelvic pain syndrome (CP/CPPS) is a complex condition, characterized by uncertain etiology and by limited response to therapy. The definition of CP/CPPS includes genitourinary pain with or without voiding symptoms in the absence of uropathogenic bacteria, as detected by standard microbiological methods, or another identifiable cause such as malignancy. The efficacy of various medical therapies, has been evaluated in clinical studies, but evidence is lacking or conflicting. We compared Serenoa Repens in monotherapy versus Palmitoylethanolamide (PEA) in combination with Alpha-lipoic acid (ALA) and evaluated the efficacy of these treatments in patients with CP/CPPS.

Methods: We conducted a randomized, single-blind trial. 44 patients diagnosed with CP/CPPS (mean age $41.32 \pm 1.686$ years) were randomly assigned to treatment with Palmitoylethanolamide $300 \mathrm{mg}$ plus Alpha-lipoic acid $300 \mathrm{mg}$ (Peanase $^{\circledR}$ ), or Serenoa Repens at $320 \mathrm{mg}$. Three questionnaires (NIH-CPSI, IPSS and IIEF5) were administered at baseline and after 12 weeks of treatment in each group. Results: 12 week treatment with Peanase significantly improved the IPSS score compared to the same period of treatment with Serenoa Repens, and significantly reduced NIH-CPSI score. Similar results were observed in the different NIH-CPSI subscores break down. However, the same treatment did not result in significant improvement of the IIEF5 score. Both treatments did not produce undesired effects. Conclusions: The present results document the efficacy of an association of Palmitoylethanolamide (PEA) and Alpha-lipoic acid (ALA) administered for 12 weeks for treating patients with CP/CPPS, compared with Serenoa Repens monotherapy.
\end{abstract}

KEY WORDS: Chronic pelvic pain syndrome; Chronic prostatitis; Palmitoylethanolamide; Serenoa repens; Lipoic acid.

Submitted 14 May 2016; Accepted 17 July 2016

\begin{abstract}
INTRODUCTION
Chronic prostatitis/chronic pelvic pain syndrome (CP/CPPS) is a complex condition, characterized by uncertain etiology and by limited response to therapy. The definition of CP/CPPS includes genitourinary pain with or without voiding symptoms in the absence of uropathogenic bacteria, as detected by standard microbi-
\end{abstract}

ological methods, or another identifiable cause such as malignancy. CP/CPPS is a highly prevalent condition affecting males of a wide age range, and can significantly impair the quality of life (QoL) and the social functioning of patients. Lifetime CP/CPPS prevalence is $2 \%$ to $10 \%$. Many etiologies and mechanisms for CP/CPPS pathogenesis have been proposed, including infection, detrusorsphincter dysfunction, immunological dysfunction, interstitial cystitis, and neuropathic pain. CP/CPPS is characterized by a wide spectrum of symptoms, including chronic or persistent pain in the pelvic region, irritative and obstructive voiding symptoms, ejaculatory pain, sexual dysfunction, depression and psycho-social maladjustment amongst others. The CP/CPP mechanisms involve ongoing acute pain mechanisms (such as those associated with inflammation or infection) and chronic pain mechanisms (such as central sensitization or visceral hyperalgesia) (1). The efficacy of various medical therapies, such as antibiotics, alpha-adrenergic blockers, anti-inflammatory agents, hormonal therapies, and phytotherapies, has been evaluated in clinical studies of CPPS. However, evidence is lacking or conflicting. Phytotherapeutics are a noteworthy option due to their generally minimal side-effects; however, few have been subjected to scientific scrutiny and prospective controlled clinical trials.

It has been hypothesized that the etiological determinants of CP/CPPS are likely to trigger tissue and cellular responses that include inflammation and the upregulation of cytokine expression and release. Inflammatory injury may damage tissue components such as nerves and blood vessels, in turn causing pain that may produce contraction of pelvic smooth and skeletal muscles, finally leading to lower urinary tract symptoms, ejaculatory pain and pain in other regions, including the lower back and abdomen. Prolonged pain may lead to neurogenic inflammation and peripheral and central sensitization.

\section{Patients And methods}

CP/CPPS is treated by means of different protocols. We compared Serenoa Repens in monotherapy versus Palmitoylethanolamide (PEA) in combination with 
Alpha-lipoic acid (ALA) and evaluated the efficacy of these treatments in patients with CP/CPPS. We conducted a randomized, single-blind trial of a community population recruited from June 2014 to January 2015.

\section{Trial design}

Men aged between 22 and 61 years, attending our outpatient urology clinic were invited to participate in the study. Inclusion criteria were: complaints of chronic pelvic pain for at least six months; IPSS score higher than 13 at visit 1; pain domain of NIH-CPSI higher than 1 at visit 1 ; total PSA lower than $4 \mathrm{ng} / \mathrm{ml}$. Patients were included in this study if they exhibited at a first visit signs and symptoms of category III CP/CPPS, according to National Institutes of Health (NIH) criteria (NIDDK Chronic Prostatitis Workshop, 1995). Subjects younger than 22 and older than 61 years, affected by major comorbidities, with known anatomical abnormalities of the urinary tract or with evidence of other urological diseases, and with residual urine volume $>50 \mathrm{ml}$ resulting from bladder outlet obstruction were excluded. Males with a reported allergy to the drugs administered during the trial, who had recently $(<4$ weeks) undergone oral or parenteral treatment or who were currently using prophylactic antibiotic drugs and/or finasteride were also excluded. All patients positive to tests for Chlamydia trachomatis (Ct), Ureaplasma urealyticum, Neisseria gonorrhoeae, herpes viruses (HSV 1/2) and human papillomavirus (HPV) were also excluded.

The severity of the chronic prostatitis symptoms was scored by means of an Italian validated version of the NIH Chronic Prostatitis Symptom Index (NIH-CPSI), addressing pain and voiding symptoms, and the impact of the disease on patients' quality of life (QoL). The International Index of Erectile Function (IIEF) questionnaire was adopted to assess the erectile function of patients. Mild to severe erectile dysfunction (ED) was defined as the sum of the scores for IIEF questions 1-5 and 15 , which in total were inferior to 26. The IPSS is a validated, seven-item questionnaire evaluating LUTS occurring during the preceding month, with lower scores indicating less severe LUTS.

Forty-four patients diagnosed with CPPS were randomly assigned to treatment with Palmitoylethanolamide 300 mg plus Alpha-lipoic acid 300 mg (Peanase $\left.{ }^{\circledR}\right)$, two capsules every 24h (group A), or Serenoa Repens at $320 \mathrm{mg}$, one capsule every 24h (group B).

NIH-CPSI, IIEF, and IPSS questionnaires were completed by each patient prior to and 12 weeks after the treatment. Mean values of total scores before and following the treatment were compared. At time-point V2 (visit two), after complete clinical and microbiological assessments, patients received a full course of pharmacological therapy. Microbiological eradication of pathogens was assessed before enrolment. At time-point V3 (visit three), performed 12 weeks after the start of therapy, symptoms were assessed again with questionnaires. Data are presented as means \pm standard error. Changes from baseline within each group were assessed through one-way analysis of variance (one-way ANOVA), followed by a Bonferroni test (Bonferroni's multiple comparison test). Statistical significance was assessed at a p value $<0.05$. The full analysis set included patients who received 84 doses of the study drugs. It was estimated that 22 patients per treatment group were required to demonstrate efficacy in terms of symptoms score changes from baseline. The safety analysis consisted of all randomised subjects who took one dose or more of study medication.

\section{Results}

In this study 44 patients were enrolled and randomised into two groups (mean age $41.32 \pm 1.686$ years). Among them, 22 received Peanase ${ }^{\circledR}$ (palmitoylethanolamide plus alpha-lipoic acid), group A, and 22 received Serenoa Repens, group B. The treatment arms were comparable for all variables at the enrollment and randomisation visits. Three questionnaires (NIH-CPSI, IPSS and IIEF5 scores) were administered at baseline and after 12 weeks of treatment in each group.

Data indicate that 12 week treatment with Peanase significantly improved the IPSS score compared to the same period of treatment with Serenoa Repens (Figure 1).

However, the same treatment did not result in significant improvement of the second score analysed, the IIEF5 (Figire 2).

Finally, the 12-week treatment with Peanase was able to significantly reduce NIH-CPSI score. Similar results were observed in the different NIH-CPSI subscores break down. On the other hand, statistical of data from patients treated with Serenoa Repens did not result in significant differences between treated and untreated patients (Figure 3).

Both treatments were well tolerated in all the patients examined, and no significant drug-related side effects were noticed.

\section{Figure 1.}

Comparative effects of 12-week treatments with either the combination á-lipoic acid-palmitoylethanolamide or the extract from S. Repens on the IPSS score for evaluation of urinary symptoms in patients with chronic prostatitis. Vertical bars are means \pm SE; ${ }^{\star} p<0.05$ vs. S. Repens (one way analysis of variance, followed by the Bonferroni's multiple comparison test).

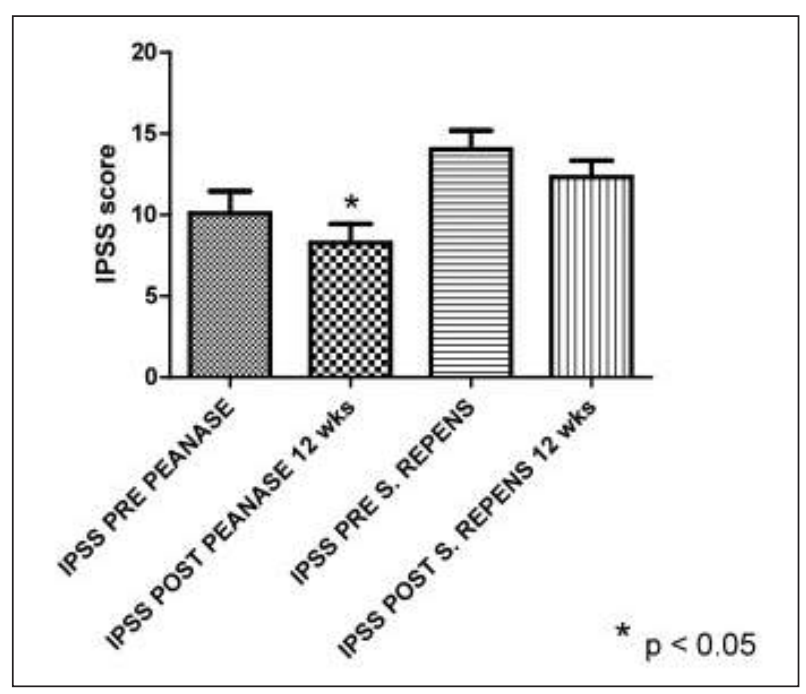




\section{Figure 2.}

Comparative effects of 12-week treatments with either the combination á-lipoic acid-palmitoylethanolamide or the extract from S. Repens on the IIEF5 score for evaluation of erectile function in patients with chronic prostatitis. Vertical bars are means \pm SE; significance was set for a ${ }^{*} p<0.05$ (one way analysis of variance, followed by the Bonferroni's multiple comparison test).

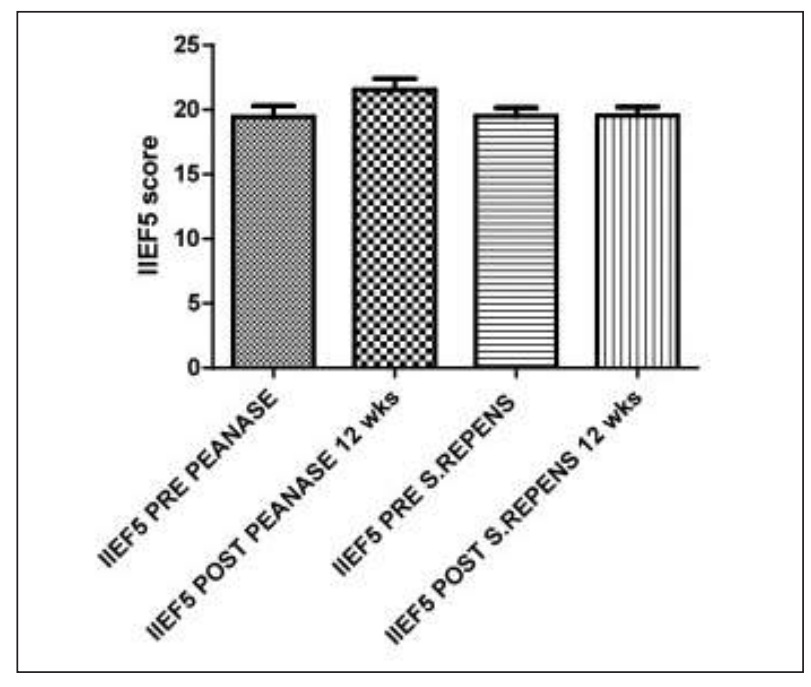

Figure 3.

Comparative effects of 12-week treatments with either the combination á-lipoic acid-palmitoylethanolamide or the extract from S. Repens on the NIH-CPSI score for evaluation of pain, urinary discomfort and relative quality of life in patients with chronic prostatitis. Vertical bars are means $\pm S E ;{ }^{*} p<0.05$ vs. time zero of the combination (one way analysis of variance, followed by the Bonferroni's multiple comparison test).

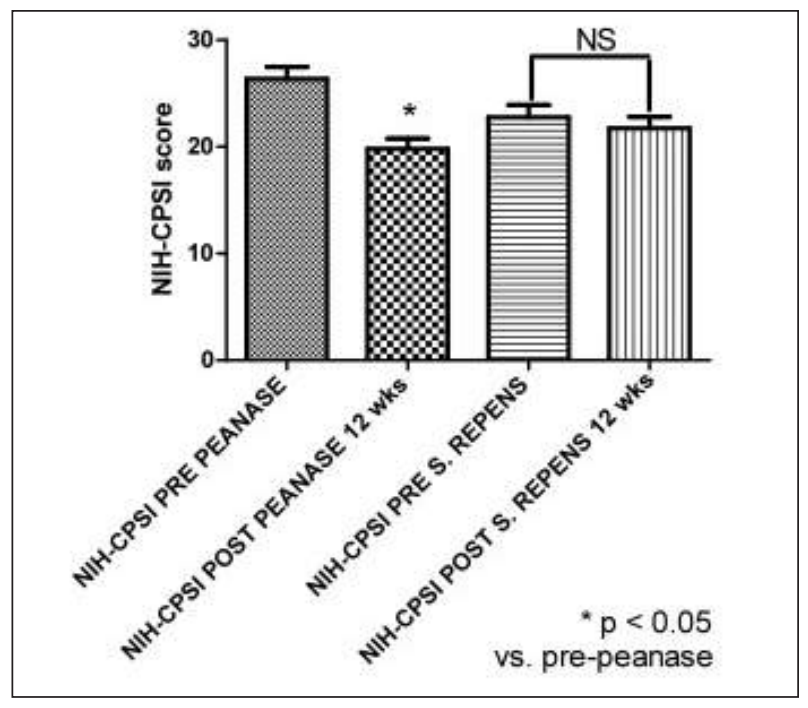

\section{Discussion}

The most recent NIH classification of prostatitis adopted in 1995 includes several clinical entities, such as acute and chronic bacterial infections, CPPS, and asymptomatic inflammation of the prostate. The most common type of prostatitis is category III, also known as CP/CPPS. The current NIH definition of CP/CPPS includes genitourinary pain with or without voiding symptoms in the absence of uropathogenic bacteria, as detected by standard microbiological methods, or another identifiable cause such as malignancy. The efficacy of various medical therapies, such as antibiotics, alpha-adrenergic blockers, anti-inflammatory agents, hormonal therapies, and phytotherapies, has been evaluated clinically and is however a matter of debate.

In this short study we demonstrate that the combination of alpha-lipoic acid with palmitoylethanolamide improves significantly parameters related to prostate function in course of inflammatory processes.

Alpha-lipoic acid (ALA), or 1,2-dithiolane-3-pentanoic acid, is a natural-occurring co-factor for vital metabolic multienzyme complexes, including pyruvate dehydrogenase and glycine decarboxylase. ALA is absorbed from the diet, and it is readily converted to its reduced form, dihydrolipoic acid, in mammalian cells. ALA acts as a redox regulator of thiolcointaining proteins and is effective in the treatment of several pathologic conditions including diabetes, neurodegeneration, radiation injury, and malignancy (2). Growing evidence shows that orally supplied ALA may not be used as a metabolic cofactor, but instead elicits a unique set of biochemical activities with potential pharmacotherapeutic value against a host of pathophysiologic insults. ALA has been described as a potent biological antioxidant, and has been implicated as a modulator of various inflammatory signalling pathways (3-4). This impressive array of cellular and molecular functions has gained considerable interest among the public and the research community for the use of ALA both as a nutritive supplement and as a pharmacotherapy.

The chemical reactivity of ALA is mainly conferred by its dithiolane ring. The oxidized (ALA) and reduced (DHLA) forms create a potent redox couple that has a standard reduction potential of $-0.32 \mathrm{~V}$. This makes DHLA one of the most potent naturally occurring antioxidants (5). In fact, there is evidence that both ALA and DHLA are capable of scavenging a variety of reactive oxygen species. In addition to being direct reactive oxygen species scavengers, both ALA and DHLA chelate redox-active metals in vitro and in vivo. The oxidized and reduced forms bind a number of metal ions, but with different properties depending on the metal chelated. These results imply, but do not yet prove, that ALA supplementation may modulate the labile pool of redox active transition metals, without causing metal depletion.

There is growing evidence that ALA may act indirectly to maintain cellular antioxidant status by either inducing the uptake or enhancing the synthesis of endogenous low molecular weight antioxidants or antioxidant enzymes. For instance, reports show that ALA increases intracellular ascorbate levels. In concert with improving ascorbate status, LA markedly increases intracellular glutathione (GSH), an abundant natural thiol antioxidant and co-substrate for detoxification enzymes, in a variety of cell types and tissues (6-7).

Several clinical trials have been conducted to measure the efficacy of racemic ALA in decreasing symptoms of diabetic polyneuropathies; these are the "alpha-lipoic acid 
in diabetic neuropathy" (ALADIN) trials and the "symptoms of diabetic polyneuropathy" (SYDNEY) trials. ALA was given orally, intravenously, or i.v. with oral follow-up. However, some additional studies have found that oral ALA is very effective. For example, the oral pilot (ORPIL) study showed a reduction in diabetic polyneuropathic symptoms after three weeks with $600 \mathrm{mg}$ ALA t.i.d. (8) While the first SYDNEY trial used i.v. LA (9), the SYDNEY II study used oral LA at 600,1200 , or $1800 \mathrm{mg}$ q.d. for 5 weeks (10); consequently, both studies showed significant improvements in neuropathic endpoints.

ALA has also been studied for its antioxidant properties in cytokine-induced inflammation; it is also widely known as an inhibitor of NF-kappaB (11). Results show that ALA lowers expression of vascular cell adhesion molecule-1 (VCAM-1) and endothelial adhesion of human monocytes, and inhibits NF-kappaB-dependent expression of metalloproteinase-9 in vitro (12-13). Similarly, ALA (25$100 \mathrm{ig} / \mathrm{ml}=122-486 \mathrm{iM}$ ) prevents the upregulation of intercellular adhesion molecule-1 (ICAM-1) and vascular cell adhesion molecule-1 (VCAM-1) in spinal cords and in TNF-alpha stimulated cultured brain endothelial cells (14). To date, the anti-inflammatory properties of ALA have rarely been investigated in humans. The ISLAND trial showed a $15 \%$ significant decrease in serum interleukin-6 levels following 4 weeks of supplementation with ALA (300 mg/day) (15).

Although some important aspects of ALA's mechanism of action in vivo are yet to be uncovered, it is apparent that oral ALA supplements are clinically effective in mitigating chronic inflammatory conditions, as well as improving or maintaining antioxidant/detoxification defenses that otherwise decline with age.

Among molecules able to modify the course of chronic pain in adults, palmitoylethanolamide (PEA) seems to deserve interest. PEA is an endogenous Nacylethanolamine produced on demand to promote the resolution of neuroinflammation and pain (16). PEA administration seems able to control reactivity of tissue peripheral mast cells located in close proximity to nerve terminals and within the endoneural microenvironment, thereby normalizing the sensitivity and function of primary somatosensory peripheral neurons (17).

Furthermore, PEA act on spinal/supraspinal nonneuronal cells (microglia, resident or infiltrating mast cells, astrocytes) to counteract neuroinflammation and normalize the activity of second- and third-order somatosensory neurons $(18,19)$.

Clinical studies demonstrate the efficacy of PEA in reducing chronic and neuropathic pain associated with various pathological conditions. A remarkable pharmacological feature of PEA is that its addition to ongoing standard therapies for chronic or neuropathic pain in patients with unsatisfactory management of pain relief allows a significant reduction of non-steroidal antiinflammatory drug use (20).

In the present study, a significant reduction of the total NIH-CPSI score was achieved in the group of patients subjected to combination therapy (ALA plus PEA) for a period of 12 weeks. Notably, this improvement in NIHCPSI score was not seen in the group of patients treated with Serenoa Repens. The same observation comes from the evaluation of IIEF score in both arms. Patient treated with ALA plus PEA showed a significant improvement in erectile function, not seen in the arm treated with Serenoa Repens.

This study clearly has limitations. The small number of subjects may not be representative of the general population with CP/CPPS. The absence of a placebo arm may be considered a limitation in terms of efficacy evaluation of administered treatments, anyway our aim was a direct comparison between a new therapeutic option for CP/CPPS and a traditional treatment strategy, rather than an absolute measurement of efficacy. Furthermore, a significant psychological component is well described in patients with CP/CPPS symptoms, and a comparable placebo effect can be assumed in all patients taking drugs to treat this kind of symptoms. More extensive studies to assess these parameters will enhance our understanding of the mechanism of action of ALA plus PEA in patients with CP/CPPS.

In conclusion, given the aforementioned limitations, the present results document the efficacy of an association of Palmitoylethanolamide (PEA) and Alpha-lipoic acid (ALA) administered for 12 weeks for treating patients with CP/CPPS, compared with Serenoa Repens monotherapy. The treatment did not produce undesired effects. However, additional long term and thorough clinical studies are needed to better explain the mechanisms of its neuroprotective effects and to further investigate the efficacy of its supplementation.

\section{REFERENCES}

1. Magri V, Marras E, Restelli A, et al. Multimodal therapy for category III chronic prostatitis/chronic pelvic pain syndrome in UPOINTS phenotyped patients. Exper Ther Med. 2015; 9:658-666.

2. Sunghyup C, Kweonsik M, Ikjoon C, et al. Effects of alpha-lipoic Acid on the antioxidant system in prostate cancer cells. Korean J Urol. 2009; 50:72-80.

3. Shay KP, Moreau, RF, Hagen TM, et al. Alpha-lipoic acid as a dietary supplement: Molecular mechanisms and therapeutic potential. Biochim Biophys Acta. 2009; 1790:1149-1160.

4. Smith AR, Shenvi SV, Widlansky M, et al. Lipoic acid as a potential therapy for chronic diseases associated with oxidative stress. Curr Med Chem. 2004; 11:1135- 46.

5. Scott BC, Aruoma OI, Evans PJ, et al. Lipoic and dihydrolipoic acids as antioxidants. A critical evaluation. Free Radic Res. 1994; 20:119-33

6. Bast A, Haenen GR. Interplay between lipoic acid and glutathione in the protection against microsomal lipid peroxidation. Biochim Biophys Acta. 1988; 963:558-61.

7. Busse E, Zimmer G, Schopohl B, et al. Influence of alpha-lipoic acid on intracellular glutathione in vitro and in vivo. Arzneimittelforschung. 1992; 42:829-31.

8. Ruhnau KJ, Meissner HP, Finn JR, et al. Effects of 3-week oral treatment with the antioxidant thioctic acid (alpha-lipoic acid) in symptomatic diabetic polyneuropathy. Diabet Med. 1999; 16:1040-3.

9. Ametov AS, Barinov A, Dyck PJ, et al. The sensory symptoms of diabetic polyneuropathy are improved with alpha-lipoic acid: the SYDNEY trial. Diabetes Care. 2003; 26:770-6. 
10. Ziegler D, Ametov A, Barinov A, et al. Oral treatment with alpha-lipoic acid improves symptomatic diabetic polyneuropathy: the SYDNEY 2 trial. Diabetes Care. 2006; 29:2365-70.

11. Packer L, Witt EH, Tritschler HJ. alpha-Lipoic acid as a biological antioxidant. Free Radic Biol Med. 1995; 19:227-50.

12. Kunt T, Forst T, Wilhelm A, et al. Alpha-lipoic acid reduces expression of vascular cell adhesion molecule-1 and endothelial adhesion of human monocytes after stimulation with advanced glycation end products. Clin Sci (Lond). 1999; 96:75-82.

13. Kim HS, Kim HJ, Park KG, et al. Alpha-lipoic acid inhibits matrix metalloproteinase-9 expression by inhibiting NF-kappaB transcriptional activity. Exp Mol Med. 2007; 39:106-13.

14. Chaudhary P, Marracci GH, Bourdette DN. Lipoic acid inhibits expression of ICAM-1 and VCAM-1 by CNS endothelial cells and T cell migration into the spinal cord in experimental autoimmune encephalomyelitis. J Neuroimmunol. 2006; 175:87-96.

15. Sola S, Mir MQ, Cheema FA, et al. Irbesartan and lipoic acid improve endothelial function and reduce markers of inflammation in the metabolic syndrome: results of the Irbesartan and Lipoic Acid in
Endothelial Dysfunction (ISLAND) study. Circulation. 2005; 111:343-8.

16. Paladini A, Fusco M, Coaccioli S, et al. Chronic pain in the elderly: the case for new therapeutic strategies. Pain Physician. 2015; 18:E863-E876

17. Bettoni I, Comelli F, Colombo A, et al. Non-neural cell modulation relieves neuropathic pain: efficacy of the endogenous lipid palmitoylethanolamide. CNS Neurol Disord Drug Targets. 2013; 12:34-44.

18. Luongo L, Guida F, Boccella S, et al. Palmitoylethanolamide reduces formalininduced neuropathic-like behaviour through spinal glial/microglial phenotypical changes in mice. CNS Neurol Disord Drug Targets. 2013; 12:45-54.

19. Cantarella G, Scollo M, Lempereur L, et al. Endocannabinoids inhibit release of nerve growth factor by inflammation-activated mast cells. Biochem Pharmacol. 2011; 82:380-388.

20. Dominguez CM, Martín A, Ferrer FG, et al. N-palmitoylethanolamide in the treatment of neuropathic pain associated with lumbosciatica. Pain Manage. 2012; 2:119-124.

\section{Correspondence}

Bruno Giammusso, MD (Corresponding Author)

bgiammusso@hotmail.it

Policlinico Morgagni, Via Vivante, 3 - 95123 Catania, Italy

Rosaria Di Mauro

rosariadimauro@tiscali

BIOMETEC, Section of Pharmacology, University of Catania School

of Medicine, Catania, Italy

Renato Bernardini

renato.bernardini@gmail.com

BIOMETEC, Section of Pharmacology, University of Catania School

of Medicine, Catania, Italy 